\title{
NEU +++ NEU +++ NEU +++ NEU
}

\section{Liebe Kolleginnen und Kollegen,}

das neue Jahr ist schon wieder in vollem Gang. Im Februar trafen sich die Referenten der Kurse 2018 zu einem Briefing in Berlin. Neben dem gemütlichen Beisammensein und dem kollegialen Austausch über die Lehrerfahrungen aus 2017 waren die Vorbereitung auf die Kurse 2018 und die Überprüfung der schriftlichen Prüfungsfragen für das A-Diplom Gegenstand des Treffens. Arbeitsgruppen beschäftigten sich mit neuen Lehrinhalten und den Prüfungsregelungen für das A-Diplom, STH- und Laser-Diplom. Neue Kurse, wie der Traumatologie-Kurs und der 2-tägige Master Tung Kurs, wurden vorgestellt.

\section{Neue Weiterbildungsordnung}

Alle Kollegen, die noch ohne einen Abschluss in der Akupunktur sind, sollten überlegen, ob sie nicht den RefresherKurs in Garmisch-Partenkirchen nutzen, um wieder einzusteigen und um die Ausbildung 2018/2019 noch abzuschließen. Die neue WBO wird eine 360 UE-Ausbildung für die Zusatzbezeichnung vorschreiben. Sicherlich ist dies einerseits aus fachlicher Sicht richtig und dient der Gleichstellung der Akupunktur mit anderen Zusatzbezeichnungen, andererseits ist es für den Anfänger eine größere Hürde und verlangt mehr Ausdauer.

\section{Timmendorf 2018}

Für Timmendorf 2018 (09.09.-14.09.2018) haben wir dieses Jahr schon die Einladung mit der Mitgliederrechnung verschickt. Nutzen Sie den attraktiven Frühbucherrabatt, der am 31.03.2018 endet. Das abwechslungsreiche Programm wird ganz verschiedene Höhepunkte haben. Der durch das Programm führende rote Faden ist dieses Jahr das Feuer-Element.

\section{Personelles}

Frau B. Mack verlässt nach über 7-jähriger Tätigkeit Ende März die DAA e.V., um sich einem neuen Berufsfeld zu widmen. Wir alle haben ihre zuverlässige und freundliche
Art geschätzt und bedauern ihren Entschluss sehr. Trotzdem sindVeränderungen Teil des Lebens, und so wünschen wir ihr viel Freude an dem neuen Aufgabenfeld.

\section{Die DAA-B e.V. informiert:}

\section{Garmisch 2018}

Nutzen Sie die Gelegenheit, sich mit einem Abschluss (ADiplom/TCM-Diplom) zu qualifizieren. Die Akupunktur erfreut sich ungebremster Beliebtheit bei Patienten. Die für 2018 erwartete neue GOÄ (Gebührenordnung für Ärzte) bildet das gesamte Spektrum der Akupunktur und weiterer Verfahren der TCM in noch nie dagewesener Weise sehr gut ab. Darunter fallen auch die IGel-Leistungen Ohrakupunktur und Störherddiagnostik. Für viele Praxen bleiben IGel-Leistungen eine wichtige wirtschaftliche Säule.

Die Diplomprüfungen können Sie vom 10.-16. Mai 2018 in Garmisch ablegen. Weitere Informationen erhalten Sie über das Vereinsbüro.

\section{Veranstaltungsticket}

Als Besucher unserer Kurse und Kongresse haben Sie ab sofort die Möglichkeit, das Veranstaltungsticket der Deutschen Bahn zum exklusiven Festpreis deutschlandweit zu nutzen. Über unsere Internetseite www.akupunktur-seminare.de (Button am Ende der Seite) haben Sie Zugriff auf das Angebot mit Best-Preis-Vergleich. Nutzen Sie die Reisezeit zumVor- oder Nachbereiten unserer Kurse, oder entspannen und genießen Sie einfach Ihre Reise nach Praxisalltag und Kurswochenende.

Ich wünsche Ihnen viel Freude an unserem Kursangebot 2018 und viel Zeit für kollegialen Austausch im Zeichen der Akupunktur

Ihr

B. Ramme

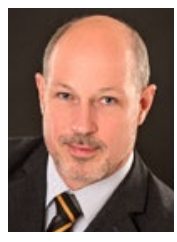

Dr. med. Bernd Ramme

1. Vorsitzender der DAA e.V.,

Osserstraße 40, D-81679 München

Tel. $+4989 / 8145252$

E-Mail des allg.Büros: kontakt@akupunktur.de, Internet:www.akupunktur.de 